\title{
Effect of bagging on the aroma compounds in mangoes during different postharvest stages
}

\author{
Changbin Wei ${ }^{1}$, Zhiling Ma ${ }^{1}$, Jian Qiao ${ }^{1}$, Xinming Tang ${ }^{1}$, Qingze Yan $^{2}$, Lizhu Tang ${ }^{2}$ and Liqing Du ${ }^{1 *}$ \\ ${ }^{1}$ Key laboratory of Tropical Fruit Biology, Ministry of Agriculture and Rural Affairs, South Subtropical Crop Research Institute, \\ Chinese Academy of Tropical Agricultural Sciences, Zhanjiang, Guangdong, 524091, China \\ ${ }^{2}$ College of Tropical Crops, Yunnan Agricultural University, Pu'er, 665000, China
}

\begin{abstract}
In order to study the effect of bagging on the aroma quality of mangoes, experiments were performed on "Tainong No.1" mangoes with 2 types of bags, namely white single-layer bags (WB) and yellow/black double-layer bag (BB). With non-bagging fruits as the control group, the aroma compounds in the mangoes in different postharvest stages were analyzed via solid phase microextraction-gas chromatography-mass spectrometry (SPME-GC-MS). The results indicated that 19 aroma compounds were detected in testing fruits, which were mainly terpenoids. We observed that the effect of bagging on the synthesis of aroma compounds in the mangoes differed among different postharvest stages. Compared with the control group, bagging inhibited the aroma synthesis in the fruits in the early postharvest stages, but facilitated it in the later stages. Additionally, the white single-layer fruit bags had a better effect on the aroma synthesis in postharvest mangoes; hence, it is advisable to use white single-layer fruit bags for mango production.
\end{abstract}

\section{Introduction}

Fruit bagging is an important practice to optimize green production in the modern fruit tree industry. This technology diminishes the irritation and damage from external environment to the fruit surface, reduces the impacts of diseases and insects on fruits and the pesticide residues, and improves the appearance quality and the commodity property of the fruits[1-3].

For many years, fruit bagging has been widely used with various fruit trees, such as those of apple [1], pear [2], grape [3], peach [4], kiwi [5], and mango [6]. Mango, an essential tropical fruit, often called as "the king of tropical fruits", has a high economic value. Research on bagging of mangoes has focused on the effect of yellow/black double-layer bag and white single-layer bag on the skin coloration of the "Irwin" mango [7], and the effect of yellow single-layer bags and non-woven bags on the appearance, quality, sugar-content, acid-content, and coloration of the "Zihua" mango [8]. However, there are very few reports on the effect of bagging on the aroma quality of mangoes. Therefore, using "Tainong No. 1 " as a model, we investigated the effect of white singlelayer bags versus yellow/black double-layer bags on the aroma quality of mangoes, in order to uncover the role of bagging on the quality of mangoes and to provide theoretical guidance on the production practice.

\section{Materials and methods}

\subsection{Experimental materials}

The selected mango variety was "Tainong No. 1", and the study was conducted at Tiandong, Baise, Guangxi Zhuang Autonomous Region. The materials used for bagging were specialized bags produced by our institute-the white single-layer bags and the yellow/black double-layer bags.

\subsection{Experimental design}

The study was designed as a single-factor random test with three treatment arms: control group (CK, no bagging), white single-layer bag (WB) group, and yellow/black double-layer bag (BB) group. Each treatment was repeated three times on three different trees. On April 5, 2018, fruits of a uniform size were selected, after approximately 40 days of flowering, and bagged after being sprayed with fungicide. Thereafter, the fruits were harvested on July 12, and samples were taken every three days at a temperature of $26^{\circ} \mathrm{C}$ for analysis.

\subsection{Experimental methods}


The method of Wei et al [9] was referred, with slight modification. The testing pulp was chopped and uniformly mixed. A total of $11 \mathrm{~g}$ mixture was weighed and put into a $20 \mathrm{ml}$ headspace sample bottle, which accounted for approximately half of the bottle volume. The microextraction was performed for $40 \mathrm{~min}$ with 100 $\mu \mathrm{m}$ Polydimethylsiloxane (PDMS) solid phase microextraction head at room temperature.

Gas chromatography-mass spectrometry analysis: Agilent $6890 \mathrm{~N}-5975$ gas chromatograph - mass spectrometer.

Chromatographic conditions: DB-5 capillary column $(30 \mathrm{~m} \times 0.25 \mathrm{~mm} \times 0.25 \mu \mathrm{m})$ was used. The column temperature was programmed: the initial temperature was maintained at $50^{\circ} \mathrm{C}$ for 1 minute, raised to $140^{\circ} \mathrm{C}$ at the rate of $5^{\circ} \mathrm{C} / \mathrm{min}$, then raised to $250^{\circ} \mathrm{C}$ at the rate of $10^{\circ} \mathrm{C} / \mathrm{min}$, and eventually maintained at $250^{\circ} \mathrm{C}$ for 10 minutes. Sample was injected via a splitless injection at an inlet temperature of $240{ }^{\circ} \mathrm{C}$, with helium as the carrier gas, at a flow rate of $1.0 \mathrm{ml} / \mathrm{min}$. The extraction head was desorbed at $240^{\circ} \mathrm{C}$ for three minutes at the injection port.

Mass spectrometry conditions: Electron impact ion source (EI); detector voltage $350 \mathrm{~V}$; ion source temperature $200^{\circ} \mathrm{C}$; interface temperature $250^{\circ} \mathrm{C}$, electron energy $70 \mathrm{eV}$; scanning mass range 35 335 amu.

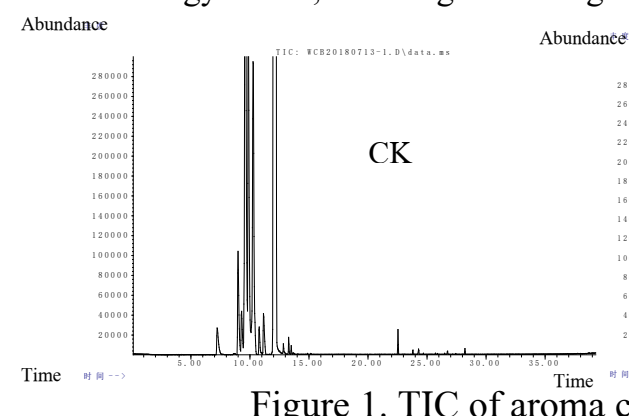

bundancer

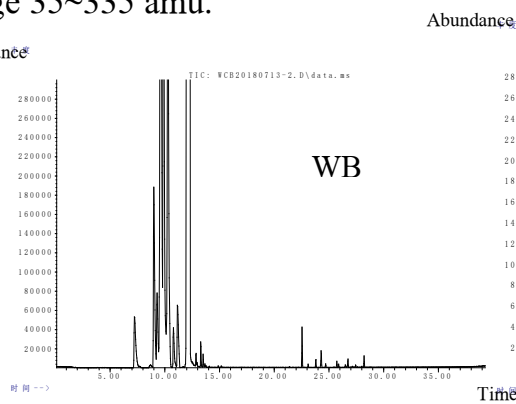

Environmental conditions: room temperature $26^{\circ} \mathrm{C}$, relative humidity $59 \%$.

\section{Results and discussion}

\subsection{Total ion chromatogram (TIC) of mango aroma compounds}

SPME-GC-MS was used to analyze the aroma compounds in mangoes of the three treatment groups (CK, WB, and BB) 1, 4, and 7 days after the harvest. The TICs are shown in Figure 1- 3.

\subsection{Effect of bagging on the aroma compounds in the mangoes.}

Aroma is one of the desirable characteristics of mangoes, and terpenoids are the main aroma compounds in these fruits [10-12]. As indicated in Table 1, 19 aroma compounds - mainly terpenoids - were detected in the mangoes on day 1 after the harvest. Of the 19 compounds, 10 were

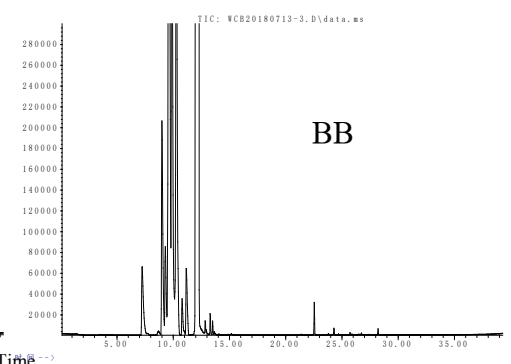

Abundance

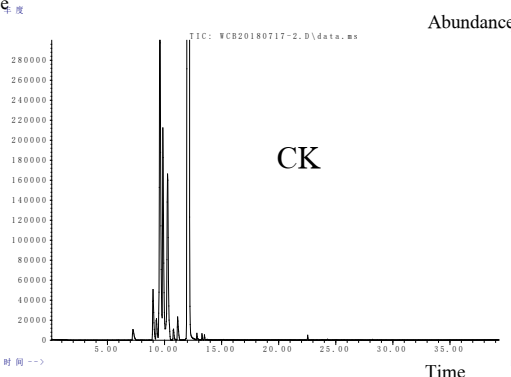

Abundance

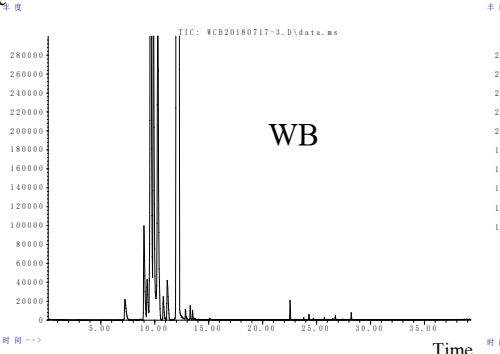

Abundance

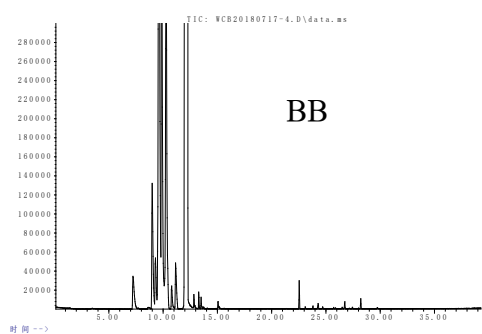

Figure 2. TIC of aroma compounds in the mangoes on day 4 after the harvest
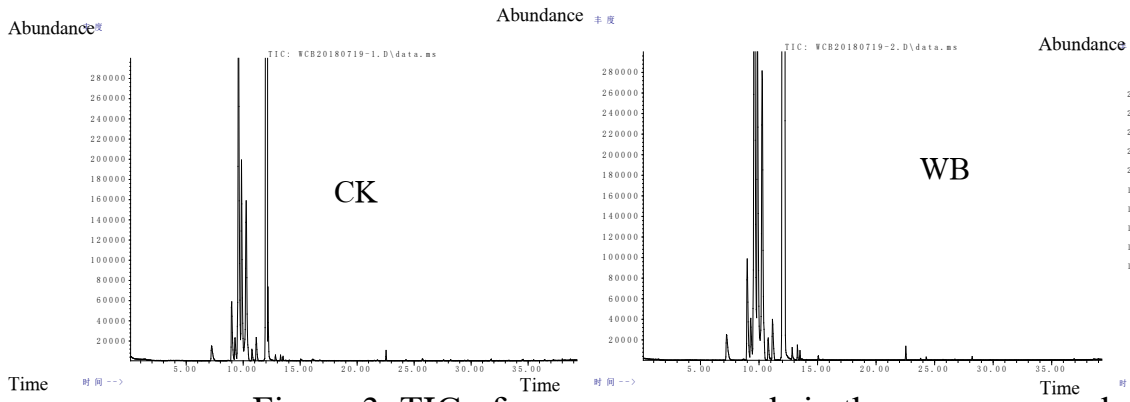

Abundance

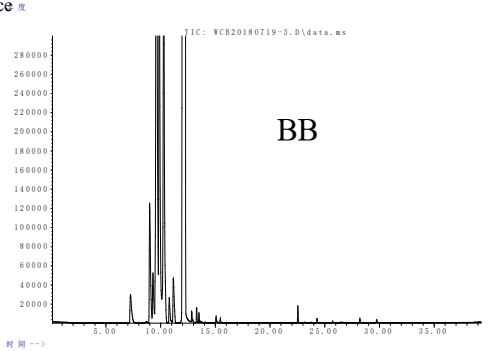

Figure 3. TIC of aroma compounds in the mangoes on day 7 after the harvest 
detected in the $\mathrm{CK}$ and $\mathrm{WB}$ groups, while 9 were detected in the BB group; the overall content of the compounds identified in the three treatment-groups were $99.10 \%, 98.84 \%$, and $98.85 \%$, respectively. On day 4 after the harvest, 5, 8, and 9 species were detected in the $\mathrm{CK}, \mathrm{WB}$, and $\mathrm{BB}$ groups, respectively, with total contents of the identified compounds as $98.82 \%, 94.82 \%$, and $99.59 \%$, respectively. On day 7 after the harvest, 7, 9, and 7 species were detected in the $\mathrm{CK}, \mathrm{WB}$, and $\mathrm{BB}$ groups, respectively, with total contents of the identified compounds as $95.86 \%, 99.12 \%$, and $96.04 \%$, respectively. These results suggest that the effect of bagging on content of the aroma compounds of mangoes was relatively small.

The common compounds on day 1 after the harvest were (Z)- $\beta$-Ocimene, $\gamma$-Terpinene, (1R)- $(+)-\alpha$-pinene, Copaene, and 4-methyl-3-(1-methylethylidene)Cyclohexene. The common compounds on day 4 after the harvest were $\gamma$-Terpinene and 4-Carene, and the common compounds on day 7 after the harvest were, (Z)$\beta$-Ocimene, (1R)- $(+)-\alpha-$ pinene, and Cinene.

\subsection{Effect of bagging on the aroma contents of mangoes}

In this study, we observed that (+)-4-Carene was detected in the CK group on day 1 after the harvest, in the BB group on day 4 after the harvest, and in both, the
CK and the BB group on day 7 after the harvest. (+)-4Carene decreased in the BB group from day 4 to day 7 . $\gamma$-Terpinene aroma content was highest in the BB group and lowest in the CK group on day 1 after the harvest, highest in the CK group on day 4 after the harvest, and highest in the BB group on day 7 after the harvest. $\gamma$ Terpinene content in the CK group increased from day 1 to day 4 , and decreased at first and then increased from day 1 to day 7 in the WB and the BB group. 4-Carene content in the $\mathrm{CK}$ and the WB group appeared to show a trend of first decreasing and then increasing from day 1 to day 7, while it was undetected in the $\mathrm{CK}$ and the $\mathrm{BB}$ group on day 7. $\alpha$-Terpinene was not detected in the WB group at any stage after the harvest, but its content increased from day 4 to day 7 in the $\mathrm{CK}$ and the $\mathrm{BB}$ group.

On day 1 after the harvest, (+)-4-Carene had the highest content among all the compounds in the $\mathrm{CK}$ group (86.58\%), and Cinene had the highest content in the $\mathrm{WB}$ and the BB group $(85.35 \%$ and $84.13 \%$, respectively). On day 4 after the harvest, (+)-4-Carene had the highest content among all the compounds in the BB group (87.78\%), and Cinene had the highest content in the CK and the WB group $(89.47 \%$ and $89.37 \%$, respectively). On day 7 after the harvest, (+)-4-Carene had the highest content in the $\mathrm{CK}$ and the $\mathrm{BB}$ group ( $85.71 \%$ and $85.89 \%$, respectively), and Cinene had the highest content in the in WB group (86.46\%).

$\underline{\text { Table 1. Content of aroma compounds in mangoes in different postharvest stages with different bags }}$

\begin{tabular}{|c|c|c|c|c|c|c|c|c|c|c|}
\hline \multirow{2}{*}{ No. } & \multirow{2}{*}{ Compounds } & \multicolumn{3}{|c|}{ 1d } & \multicolumn{3}{|c|}{$4 \mathrm{~d}$} & \multicolumn{3}{|c|}{$7 \mathrm{~d}$} \\
\hline & & $\mathrm{CK}$ & WB & BB & $\mathrm{CK}$ & WB & BB & $\mathrm{CK}$ & WB & $\mathrm{BB}$ \\
\hline 1 & (+)-4-Carene & 86.58 & & & & & 87.78 & 85.71 & & 85.89 \\
\hline 2 & (Z)- $\beta$-Ocimene & 0.18 & 0.19 & 0.16 & & 0.13 & 0.13 & 0.13 & 0.16 & 0.16 \\
\hline 3 & $\gamma$-Terpinene & 0.31 & 3.14 & 9.88 & 2.32 & 0.26 & 0.28 & & 3.17 & 6.38 \\
\hline 4 & Germacrene D & 2.7 & 0.02 & & & & & & & \\
\hline 5 & $\alpha$-Gurjunene & & & & & 0.05 & & & & \\
\hline 6 & (1R)-(+)- $\alpha$-pinene & 0.28 & 0.34 & 0.78 & & 0.18 & 2.82 & 0.31 & 0.29 & 0.28 \\
\hline 7 & 3,4-Dimethyl-2,4,6-octatriene & & & & & & & & & \\
\hline 8 & (3E,5E)-2,6-Dimethyl-1,3,5,7-octatetrene & & 0.06 & 0.06 & & & & & 0.06 & \\
\hline 9 & 4-Carene & 5.87 & 6.3 & & 4.57 & 4.73 & 5.51 & 6.22 & 5.96 & \\
\hline 10 & Copaene & 0.08 & 0.08 & 0.06 & & 0.05 & 0.07 & & 0.05 & 0.05 \\
\hline 11 & $\alpha$-Terpinene & 2.71 & & & 2.23 & & 2.17 & 2.85 & & 2.93 \\
\hline 12 & Cinene & & 85.35 & 84.13 & 89.47 & 89.37 & & 0.34 & 86.46 & 0.35 \\
\hline 13 & 2-Carene & & 2.98 & 3.3 & & & & & 2.91 & \\
\hline 14 & $\beta$-terpinene & & & & & & 0.78 & & & \\
\hline 15 & 1S-alpha.-Pinene & & & & & & & 0.3 & & \\
\hline 16 & 1,3,5,5-tetramethyl-1,3-Cyclohexadiene, & 0.06 & & & & 0.05 & 0.05 & & 0.06 & \\
\hline 17 & 4-methyl-3-(1-methylethylidene)-Cyclohexene & 0.33 & 0.37 & 0.43 & & & & & & \\
\hline 18 & 1,3-Cyclopentadiene, 1,2,3,4,5-pentamethyl- & & & 0.05 & & & & & & \\
\hline 19 & Cyclopentene, 4-ethenyl-1,5,5-trimethyl- & & & & 0.23 & & & & & \\
\hline & Number of compounds & 10 & 10 & 9 & 5 & 8 & 9 & 7 & 9 & 7 \\
\hline & Total content & 99.1 & 98.84 & 98.85 & 98.82 & 94.82 & 99.59 & 95.86 & 99.12 & 96.04 \\
\hline
\end{tabular}


It is known that fruit bags with different colors have different effects on the aroma of the fruits. A study showed that in "Kyoho" grape berries, the change in common-ester content in fruits with blue bagging was minor, while the aldehyde content increased, in comparison with the control group; in fruits with green and red bagging, the contents of esters, aldehydes, and ketones were decreased; and in fruits with white bagging, the content of esters decreased while the contents of aldehydes and ketones increased; each color bagging reduced the content of all types of alcohols but increased the types of terpenes and aromatic compounds [13]. A study conducted on "Fuji" apples showed that in fruits with red bagging, the relative contents of olefins and alcohols were high, while the relative content of esters was low; in fruits with blue bagging, the relative content of olefins was high, while the relative contents of esters and alcohols were low; and for fruits with green bagging, the relative contents of esters and alcohols were high, while the relative content of olefins was low [14]. In a study conducted on "Shine Muscat" grape berries, while the content of aldehydes in the green and the blue bagging was significantly higher than that in the white bagging [15]. Research conducted on Korla pear indicated that the composition and content of aroma compounds significantly differed among different treatments; the total content of aroma compounds in fruits with bagging was lower than that in the control group, and the content of the "fruit-flavor" esters was also significantly suppressed [16]. Research conducted on the bagging of "Abubai" peaches indicated that bagging decreased the total aroma content in fruits; it increased the alcohol content while decreased the aldehyde and ester contents of the peach fruits; moreover bagging also facilitated, the formation of acids and other compounds [17]. Overall, results of the above-mentioned studies indicate that bagging impairs the formation of the aroma compounds in fruits, and could therefore turn them flavourless.

In this study, the effect of bagging on the postharvest mango aroma was higher with white single-layer in comparison with the yellow/black bags. On day 1 after the harvest, the number of aroma compounds in the BB group was lower than that in the $\mathrm{CK}$ and the WB group, and the total aroma content in bagging treatments was lower than that in the CK group. On day 4 after the harvest, the numbers of aroma compounds in the bagging-treatment groups were higher than in the control group, and the total aroma content in the BB group was higher than in the CK group, and the total aroma content in the WB group was lower than in the CK group. On day 7 after the harvest, the total aroma contents in the bagging-treatment groups were higher than in the control group, and the number of aroma compounds in the WB group was higher than in the $\mathrm{CK}$ group. A possible explanation to these observations could be that bagging delayed the after-ripening of mango, causing the number and content of aroma compounds to become high in the late postharvest period. Alternatively, as a fruit with respiratory climacteric, the starch in the mangoes could convert to saccharides during the after-ripening of mango, and these saccharides could turn into to amino acids and fatty acids - through glycolysis and tricarboxylic acid cycle [18], respectively-which are the precursors of the aroma compounds. This could also explain why the effects of bagging on the aroma compounds in the mangoes differed between different postharvest stages. So white single-layer bags was recommended in mango production.

\section{Conclusion}

In this study, we found that 19 aroma compounds were detected and terpenoids are also the main aroma compounds of the "Tainong No. 1" mangoes. The number of aroma compounds in the BB group was lower than that in the CK and the WB group, and the total aroma content in bagging treatments was lower than that in the CK group on day 1 after the harvest. The numbers of aroma compounds in the bagging-treatment groups were higher than in the control group, and the total aroma content in the $\mathrm{BB}$ group was higher than in the CK group, and the total aroma content in the WB group was lower than in the CK group on day 4 after the harvest. On day 7 after the harvest, the total aroma contents in the bagging-treatment groups were higher than in the control group, and the number of aroma compounds in the WB group was higher than in the CK group. Compared with $\mathrm{CK}$, bagging inhibited the aroma synthesis in the fruits in the early postharvest stages, but facilitated it in the later stages. Moreover, the effect of bagging on the postharvest mango aroma was higher with white single-layer in comparison with the yellow/black bags. Based on the observations from this study, we recommend the use of white single-layer bags for bagging in mango production.

\section{Acknowledgments}

This study was supported by the grant from the Fund of Agricultural Ecological Environmental Protection of Ministry of Agriculture in 2017 and 2018; Central Public-interest Scientific Institution Basic Scientific Research Fund for Chinese Academy of Tropical Agricultural Sciences (No. $1630062016007 ; 1630062017017$; 1630062017025$)$; the Fund of Operation of Integrated Laboratory for SSCRI; the Innovation Team of Modern Agricultural Industry Technology System for Rare Fruits in Guangdong Province of China (2019KJ116); the Youth Science and Technology Talent Development Project of Guizhou Education Department(Guizhou jiao he KY [2017]322).

\section{References}

1. Li, E.M., Shi, D.C., Xu, Y.H., Chen, F., Zhai, H. (2008)Changing characteristics of temperature and humidity in different type bags for bagging apple 
and their effects on fruit appearance quality. Chinese Journal of Applied Ecology, 19(1):208-212.

2. Xu, Y.L.,Zhang, J.Y.,Yi, X.K., Gao, Z.H.,Chen, J.H. (2008)Effects of bagging on the quality of Dangshan Suli pear in Dangshan area. Journal of Anhui Agricultural University, 35(3):301-306.

3. Zhao, J.B., Luo J.X., Xiao, R.P., Wang, F, Lü, S.W. (2004)The effects of bagging on the quality grapes. Deciduous Fruits, 36(5):13-15.

4. Guo, D.h., Fan, C.h., Li, G.C. (2016)Effects of bagging on the aroma components of "Abubai" peaches. Food Science, 37(2):232-237.

5. Ma, C., Cao, S., Li, W.J., Du, J., Han, Z.C., Li, L.,Wang, R. (2019)Effects of different bagging on main quality and storability indices of 'Hongyang'kiwifruits. Food and Fermentation Industries, 45(15):202-208.

6. Zeng, K.F., Jiang, W.B. (2007)Growth Bagging Effects of Mango Fruit on Postharvest Fruit Quality and Ripening. Food Science, 28(12):507-511.

7. Ma, X.W., Xing, S.S., Wang, S.B., Yao, Q.S., Zhan, R.L., Wu, H.X., Ma, W.H. (2011)Effects of Bagging on the Pericarpial Coloration of 'Irwin' Mango During Fruit Development. Chinese Journal of Tropical Crops, 32(7):1206-1208.

8. Zeng, K.F., Jiang, W.B. (2007)Growth Bagging Effects of Mango Fruit on Postharvest Fruit Quality and Ripening. Food Science, 28(12):507-511.

9. Wei, C.B., Xing, S.S., Liu, S.H., Wu, H.X., Wang, S.B., Zang, X.P., Ma, W.H. (2010)GC-MS Analysis of Volatile Components of Mango (Mangifera indica L. var Zihua) Fruits. Food Science, 31(2):220-223.

10. Eloisa, H. A. A., Jose, G. S. M., Maria, D. G. B. Z. (2000)Aroma volatile constituents of Brazilian varieties of mango fruit. Journal of Food Composition and Analysis, 13(1):27-33.
11. Clara, E. Q., Guillermo, S., Jorge, A. P. (2007)Aroma volatile constitu-ents of colombian varieties of mango (Mangifera indica L.). Flavour and Fragrance Journal, 22(5):401-406.

12. Jorge, A. P., Judith, M. (2006)Contribution of volatile compounds to mango (Mangifera indica L.) Aroma. Flavour and Fragrance Journal, 21(2):207213.

13. Wang, H.B., Zhang, K.K., Ji X.H. (2017)Effects of different color paper bags on volatile constituents of Kyoho grape berries. Chinese Journal of Applied Ecology, 28(4):1274-1280.

14. Li Enmao, an Xiuhong, Li Min, Li Zhuang, Zhao deying, Cheng cungang. (2018)Effects of different color bags on aroma components of Fuji Apple Fruit. Journal of Fruit of Southern China, 47(5):7276.

15. Wei, Z.F., Li, Q.L., Gao, D.T., Liu, L., Liu, J.W. (2019)Effects of different colors of fruit bags on berry quality and aromatic components of 'Shine Muscat'grape. Non-wood Forest Research, 37(4):35-43.

16. Li, F.F., Zhang, H.P., He, Z.S., Tao, S.T., Li, G., Zhang, S.L. (2014)Effects of Bagging on Soluble Sugars, Organic Acids, and Aroma Compounds in Pyrus sinkiangensis'Korla Xiangli'Fruit. Acta Horticulturae Sinica, 41(7):1443-1450.

17. GUO Donghua, FAN Chonghui, LI Gaochao, et al. Effects of bagging on the aroma components of "Abubai" peaches[J]. Food Science, 2016,37(2):232-237. (in Chinese with English abstract)

18. Wang Jingyan, Zhu Shenggeng, Xu Changfa. Biological Chemistry. Beijing: Higher Edu-cation Press, 2002 (in Chinese) 